\title{
Preoperative Neoadjuvant Hormonal Therapy and Neoadjuvant Chemotherapy for Stage 3B and 4 Breast Cancer Patients in Dharmais Hospital-National Cancer Center, Indonesia: A Cohort Study
}

\author{
Ramadhan Karsono ${ }^{1}$, Denni Joko Purwanto ${ }^{1}$, Samuel J. Haryono ${ }^{1}$, Bambang \\ Karsono ${ }^{2}$, Lenny Sari ${ }^{3}$, Yulia Pratiwi ${ }^{4}$, Teguh Aryandono ${ }^{5}$ \\ ${ }^{1}$ Department of Surgical Oncology, Dharmais Hospital-National Cancer Center, Indonesia \\ ${ }^{2}$ Department of Hematology and Medical Oncology, Dharmais Hospital-National Cancer Center, Indonesia \\ ${ }^{3}$ Department of Anatomical Pathology, Dharmais Hospital-National Cancer Center, Indonesia \\ ${ }^{4}$ Department of Biostatistics, Faculty of Public Health, Universitas Indonesia, Indonesia \\ ${ }^{5}$ Department of Surgery, Faculty of Medicine Public Health and Nursing, Universitas Gajah Mada, Indonesia
}

\section{ARTICLE INFO}

\section{Article history:}

Received : 29 November 2018

Reviewed: 11 December 2018

Accepted : 10 January 2019

\section{Keywords:}

advanced breast cancer, chemotherapy, hormonal therapy, neoadjuvant

\begin{abstract}
A BSTRACT
Background: There are no data of efficacy comparison between primary systemic therapy in stage 3B and 4 breast cancer patients in Indonesia. This study compared long term outcomes of breast cancer patients treated with neoadjuvant hormonal therapy (NAHT) and those treated with neoadjuvant chemotherapy (NACT)

Methods: This was a cohort study conducted from 2011 to 2017. A total of 122 patients with stage $3 B$ and 4 breast cancer received NAHT $(n=62)$ or NACT $(n=60)$ within a 6 cycles for NACT and 6 months for NAHT were included. Patients were excluded if they had a mastectomy before treatment, were pregnant, had been given hormonal therapy or chemotherapy before, had a contra-indication of chemotherapy, had a contra-indication of salpingo-oophorectomy bilateral for premenopausal patients, and declined to enter this study. The primary outcome of this study was overall survival. The outcomes were analysed using Kaplan-Meier for survival analysis and cox proportional hazard regression to estimate the hazard ratio.
\end{abstract}

Results: There was a statistically significant difference in overall survival ( $p=0.038$ ). Median overall survival for NAHT patients was 1265 days and for NACT patients was 654 days. The hazard ratio showed NACT patients had a higher risk than NAHT patients $(1.795 \% \mathrm{Cl} 1.03-2.9)$. Pathological complete response rate was higher in the NACT group than in the NAHT group (3.3\% vs. $0 \%$ ).

Conclusions: Neoadjuvant hormonal therapy was superior to neoadjuvant chemotherapy in term of overall survival.

\section{INTRODUCTION}

Systemic therapy of breast cancer has been conducted for the last hundred years since Beatson in 1896 first demonstrated that ovarium ablation changes hormonal balancing in woman with breast cancer (1). Today, the choice of chemotherapy or hormonal therapy for breast cancer patients depends on technological advancement such as immunohistochemistry and biomolecular assay. For early breast cancer, several genomic tests have been developed to more accurately predict clinical outcomes of chemotherapy and hormonal therapy dependent on individual biological characteristics (2-4). One study with gene expression profiling showed that approximately $30 \%$ of breast cancer patients who received chemotherapy did not actually require that treatment $(2,4,5)$. However, it is different for advanced breast cancer of which the determination for using systemic therapy depends on only hormonal receptor status examination (6). 
As a care treatment, neoadjuvant systemic therapy is a standard treatment for inoperable patients (7). It offers clinical advantages to reduce tumor size, may increase the proportion of eligible patients for surgical resection or breast-conserving surgery, determine the responses to primary systemic therapy (PST) in vivo, and to determine the changes of the cells after drug administration and lead to subsequent treatment decisions (7-10). The administration of neoadjuvant systemic therapy varies from 4 cycles until 6 cycles.

The Indonesian guideline of systemic therapy for breast cancer patients still follows the guideline from Caucasian population. Thus, it may not be suitable for Indonesian population since there are notable differences between the Asian and Caucasian race based on clinical parameters, morphology and genetic factors (11). This study compared the efficacy between two types of primary systemic treatment for advanced breast cancer: neoadjuvant hormonal therapy (NAHT) and those treated with neoadjuvant chemotherapy (NACT).

\section{METHODS}

\section{Participants}

This was a prospective cohort study conducted from 2011 to 2017 at Dharmais National Cancer Center Hospital, Indonesia. Patients with stage 3B and 4 breast cancer received NAHT for 6 months or NACT within 6 cycles were included. Criteria for $3 \mathrm{~B}$ and 4 were based on American Joint Committee on Cancer 7th edition guideline (12). ER and PR status were determined by immunohistochemistry test (13). Her2 status was determined by immunohistochemistry resulting in 4 categories: Her2-negative, positive grade 1, positive grade 3 and positive grade 2 which was reconfirmed by performing FISH. Classification of histopathologic grade was based on the Nottingham Grading System. Patients were excluded if they had a mastectomy before treatment, were pregnant, declined to enter this study, had been given hormonal therapy or chemotherapy before, had a contra-indication of chemotherapy, and had contra-indication of salpingo-oophorectomy bilateral (SOB) for premenopausal patients. Patient follow up was done continuously to obtain data of death, censored patients, and patients with new symptoms.

\section{Intervention}

There were 122 patients included in this study (62 of NAHT patients and 60 of NACT patients). The study was open-label and non-randomized. Patients were assigned to one of two treatments (NAHT or NACT) by choosing the treatment based on their preferences after the advantages and disadvantages between NAHT and NACT had been explained to them. The NAHT group received Aromatase Inhibitor (AI) or Tamoxifen for postmenopausal patients and for premenopausal patients, they received Tamoxifen only or SOB and Al/Tamoxifen, or if patients rejected SOB they would receive $\mathrm{GNRH}$-analogue and $\mathrm{Al} / \mathrm{Tamoxifen}$ which was given for 6 months. Al consisted of Letrozole, Anastrozole, and Exemenestane. The NACT group received FAC (5-Fluorouracil, Adriamycin, and Cyclophosphamide) which was given for 6 cycles. After treatment, the patients were then able to receive a mastectomy when a surgically technique was possible. If not possible the patient was only able to get a second biopsy. The patient criteria for receiving a mastectomy was when the tumor was not sticking to the chest wall, not beyond the bounds of the breast and a Karnofski score higher than $90 \%$. All patients agreed to enter this study by signing the written inform consent. The flow diagram of the participants is shown in Figure 1.

This study was approved by Ethical Committee at Dharmais Hospital-National Cancer Center, Indonesia (Number of Ethic Approval: 049/PEP/08/2011) and registered in ClinicalTrials.gov (number NCT02995772).

\section{Outcomes and Statistical Methods}

The primary end point of this study was overall survival and the secondary end point was progression free survival (PFS). Overall survival was defined as the length of time from the date of pathological diagnosis until death from any cause. PFS was defined as the length of time from after treatment until tumor progression or death from any cause. Pathological complete response ( $\mathrm{pCR}$ ) was defined as the absence of residual invasive and in situ breast cancer following completion of neoadjuvant systemic therapy. In this study, pCR was defined by pathology anatomy result.

Statistical analyses were done with SPSS IBM version 21.0 and data management was done with Microsoft Excel 2010. The study used Kaplan-Meier survival analysis to produce overall survival and progression free survival. Overall survival was an output of NAHT and NACT comparison which was analyzed from survival time and events (deaths). Progressive free survival was an output of time-lapse of tumor progression after neoadjuvant systemic therapy. Hazard ratio (HR) was estimated using Cox proportional hazard regression. 


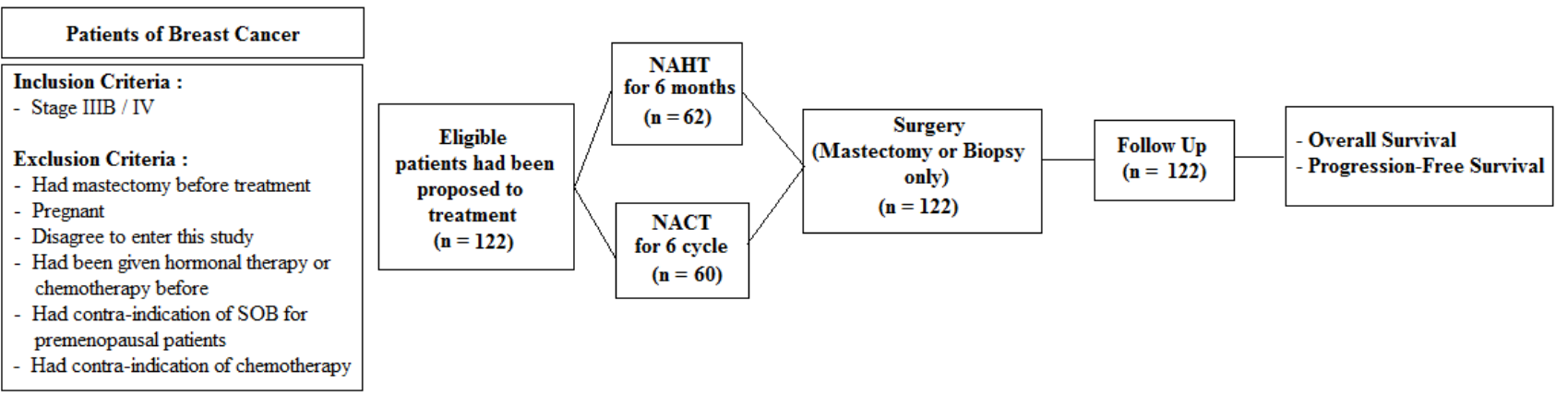

Figure 1. Research workflow

\section{RESULTS}

Table 1. Patients' characteristics

\begin{tabular}{|c|c|c|c|}
\hline Characteristics $^{\mathrm{a}}$ & NAHT $(n=62)$ & NACT $(n=60)$ & P-value $^{b}$ \\
\hline Age (year), mean (SD) & $45.7(9.3)$ & $49.8(10.5)$ & $0.023^{c}$ \\
\hline Range & $22-68$ & $27-75$ & \\
\hline Stage & & & $0.011^{d}$ \\
\hline Stage 3B & $25(39.7)$ & $38(60.3)$ & \\
\hline Stage 4 & $37(62.7)$ & $22(37,3)$ & \\
\hline Grade & & & $0.03^{d}$ \\
\hline Low Grade (I and II) & $39(60)$ & $26(40)$ & \\
\hline High Grade (III) & $23(40.4)$ & $34(59.6)$ & \\
\hline Histological Type & & & $0.54^{d}$ \\
\hline Ductal & $56(50)$ & $56(50)$ & \\
\hline Lobular & $6(60)$ & $4(40)$ & \\
\hline Hormonal Receptor & & & $<0.0001^{\mathrm{d}}$ \\
\hline Positive & $59(64.1)$ & $33(35.9)$ & \\
\hline Negative & $3(10)$ & $27(90)$ & \\
\hline Her2 Status & & & $0.25^{d}$ \\
\hline Positive $^{\mathrm{e}}$ & $13(41.9)$ & $18(58.1)$ & \\
\hline Negative & $49(53.8)$ & $42(46.2)$ & \\
\hline PCR Rate & & & $0.14^{d}$ \\
\hline $\mathrm{N}$ & 0 & 2 & \\
\hline$\%$ & 0 & 3.3 & \\
\hline Mastectomy & & & $0.44^{d}$ \\
\hline Yes & $43(48.3)$ & $46(51.7)$ & \\
\hline No & $18(56.3)$ & $14(43.8)$ & \\
\hline Metastase in Stage IV $(n=57)$ & & & $0.2^{\mathrm{d}}$ \\
\hline Visceral (lung and liver) & $16(55.2)$ & $13(44.8)$ & \\
\hline Non-visceral (bone) & $20(71.4)$ & $8(28.6)$ & \\
\hline Death/Alive & & & $0.47^{d}$ \\
\hline Death & $29(52.5)$ & $32(47.5)$ & \\
\hline Alive & $33(54.1)$ & $28(45.9)$ & \\
\hline
\end{tabular}

$\mathrm{NAHT}=$ neoadjuvant hormonal therapy; NACT= neoadjuvant chemotherapy; $\mathrm{PCR}$ rate= pathologic complete response rate; $\mathrm{SD}=$ standard deviation

${ }^{a}$ Values are number percentage

${ }^{\mathrm{b}}$ Significant value (p-value) set at 0,05 ad two tailed; ${ }^{\mathrm{C}}$ Independent sample t-test; ${ }^{\mathrm{d}}$ Pearson's Chi Square

${ }^{\mathrm{e}}$ Hormonal receptor status positive was from estrogen receptor status positive and/or progesterone receptor status positive. 
This research found the mean of patients' age of 45.7 years old \pm 9.3 years (for NAHT group) and 49.8 years old \pm 10.5 years (for NACT group). The youngest patient for NAHT and NACT group were 22 and 27 years old. The oldest patient for NAHT and NACT group were 68 and 75 years old. Pathological complete response $(\mathrm{pCR})$ rate was higher in the NACT group than in the NAHT group (3.3\% vs. $0 \%$ ). The patients characteristics in this study are shown in Table 1.

The total number of patients was 122 (62 of NAHT patients and 60 of NACT patients). There were statistical differences in OS $(p=0.038)$. Median of overall survival was 1265 days for NAHT and 654 days for NACT. The hazard ratios of overall survival by Kaplan-Meier curves (Figure 2) showed that NACT patients had higher risk of death than NAHT patients $(1.795 \% \mathrm{Cl} 1.03-2.9)$. But, there weren't statistical difference in PFS ( $p=0.65$ ) between NAHT and NACT.

There were statistical differences of overall survival by mastectomy status and hormonal receptor status. Median of overall survival was 1174 days in patients assigned to mastectomy and 587 days in nonmastectomy. The hazard ratios of overall survival by Kaplan-Meier curves (Figure 3) showed that patients that were not assigned to mastectomy had higher risk of death than patients assigned to mastectomy, and patients who had negative hormonal receptor had higher risk of death than patients who had positive hormonal receptor. Median of overall survival was 500 days for patients who had negative hormonal receptor and 1265 days for patients who had positive hormonal receptor.
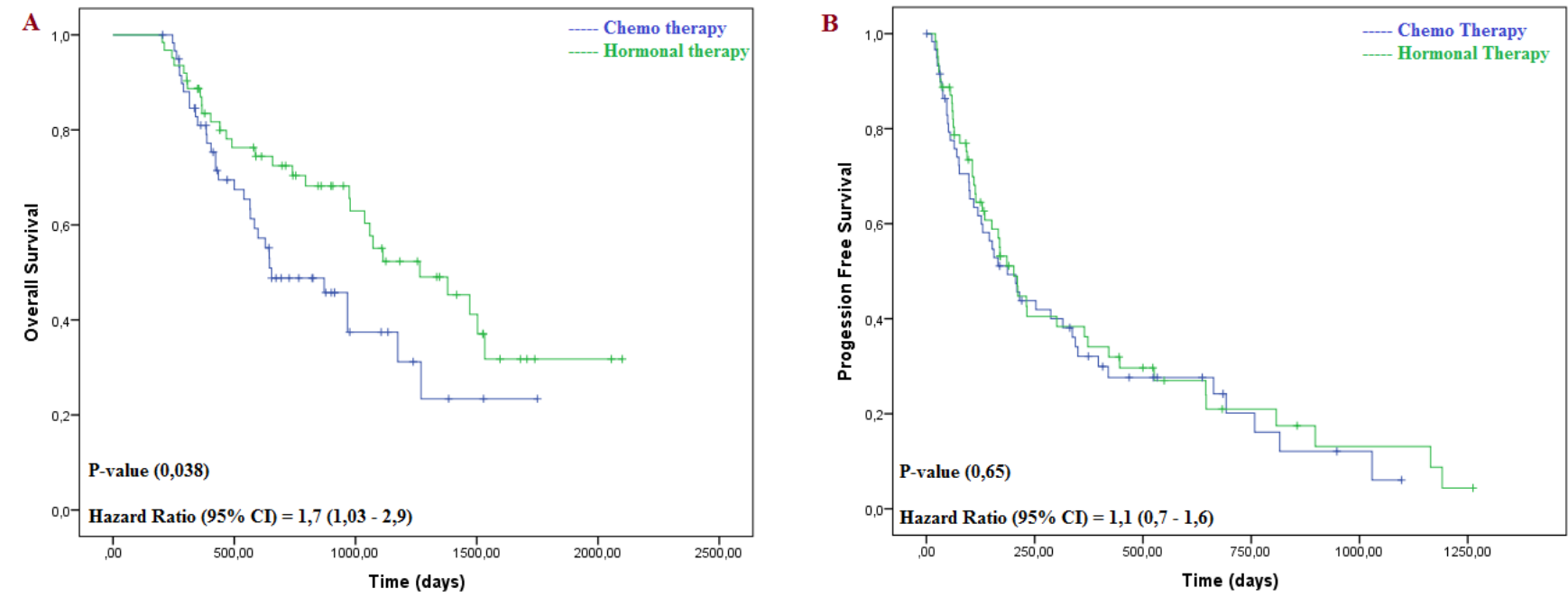

Figure 2. Kaplan-Meier curves for survival by treatment arm (A) overall survival; (B) Progression-free survival
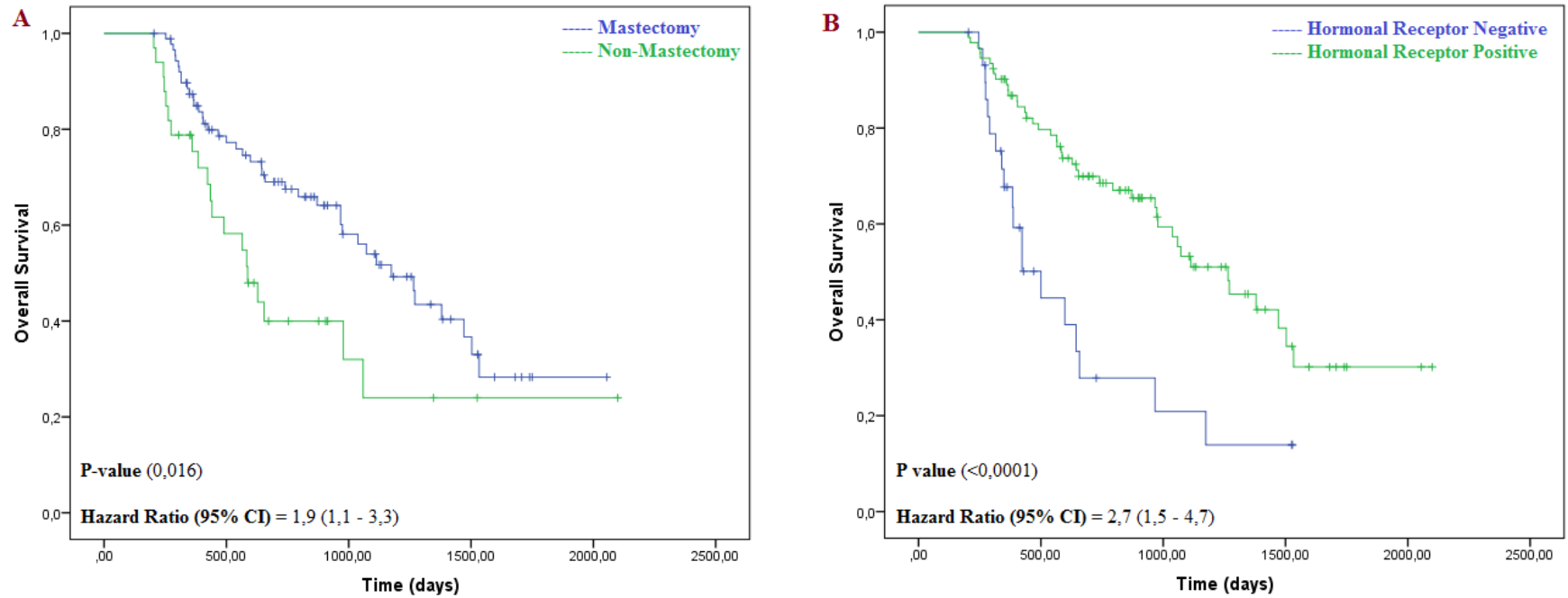

Figure 3. Kaplan-Meier curves for overall survival by (A) mastectomy status; (B) hormonal receptor status 


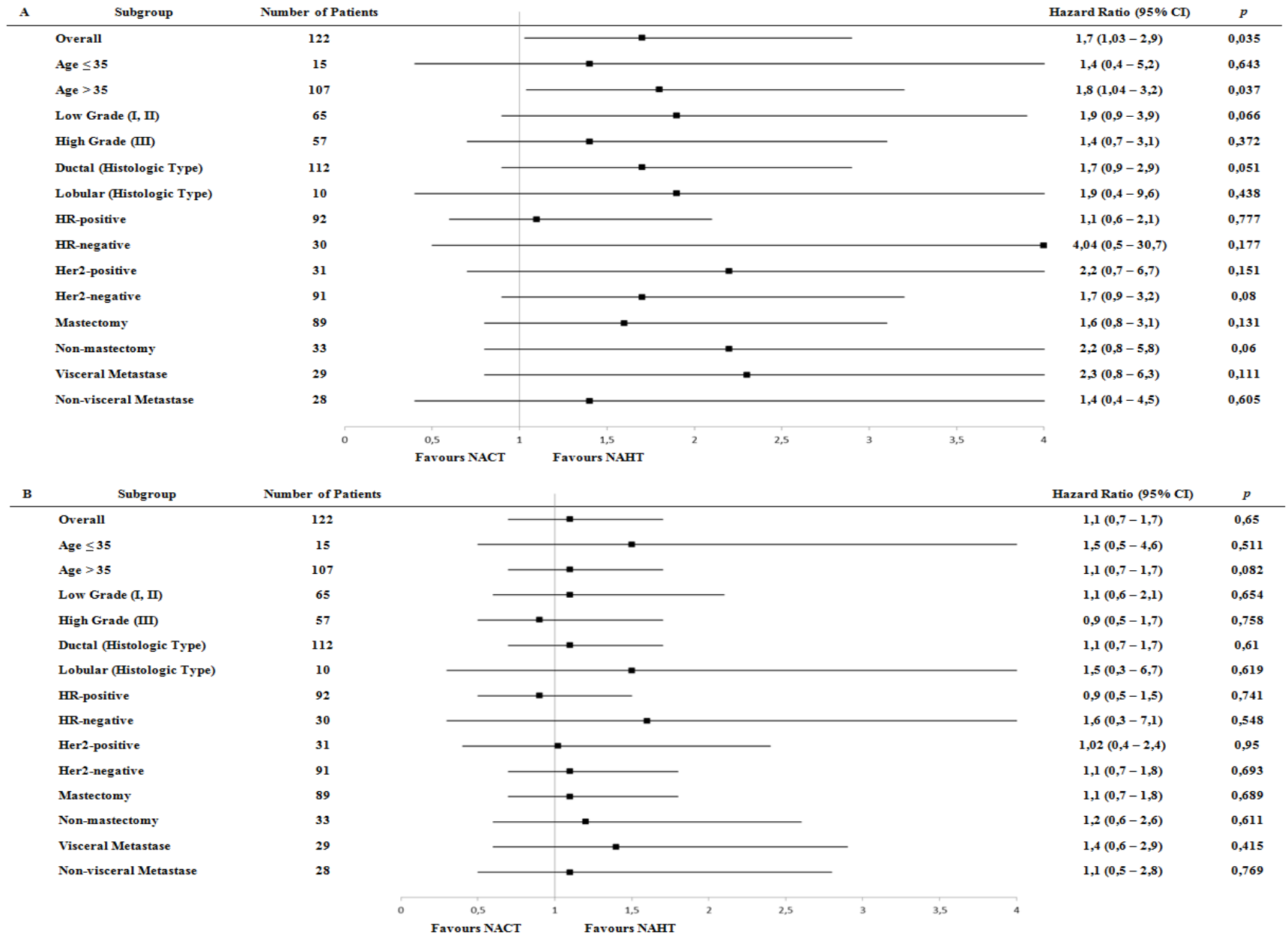

Figure 4. Forest plot of (A) overall survival, and (B) progression free survival in subgroup analysis

\section{DISCUSSION}

Findings from our study, there were statistically significant differences between NACT and NAHT in terms of age, stage, grade and hormonal receptor (Table 1). The age of patients that received NAHT is less than NACT. In older patients, there was a statistically significant difference and the Hazard Ratio was 1,8 times higher risk for death for NACT compared to NAHT (Figure 4), which is different with Marcus's study in 2013, where NACT is likely to reduce the risk of future outcomes in younger patients (14). Traditionally, NAHT is given predominantly to fragile postmenopausal patients with poor condition and NACT tends to be given to premenopausal patients to achieve a rapid response prior to surgery if technically possible (14-16).

Numerous study of neoadjuvant systemic therapy had used PCR as their endpoint. However, a common definition of $\mathrm{pCR}$ had not been defined until recently (17). Our results showed that NACT has higher pCR rate than NAHT (Table 1). The NSABP B-18 and B-27 study showed a pCR of NACT patients of $13 \%$. This rate was higher than our result because the study was conducted in early stage breast cancer patients (18). We also found patients with HR-positive, which contrast to some studies which demonstrated HR-negative tumor had higher PCR rate than HR-positive tumor $(19,20)$. Nevertheless, there were studies that concluded PCR rate could not predict survival rate $(21,22)$.

To date, there are still few studies comparing the efficacy of NACT and NAHT in breast cancer patients. Although there are such studies, generally the number of patients is small, overall survival is not frequently obtained and mostly the studies were only done on the positive hormone receptor group. In our study, we obtained statistically significant values in overall survival from comparing NAHT and NACT.

In our case, using various hormonal drugs, the results demonstrated that median survival was longer than NACT patients, median OS and PFS were 1265 days and 202 days for NAHT and 654 days and 187 days for NACT patients, with statistically significant differences (Figure 2). It is similar with other studies that showed patients who received NAHT alone had a longer median 
survival than patients who received NACT alone $(14,21,23,24)$.

In this study, $75.4 \%$ patients were hormone receptor (HR) positive. HR is a good predictor of outcomes with neoadjuvant hormonal therapy. Earlier, HR was classified with a cut-off point of $10 \%$ to be able to response to hormonal therapy. Until now, there is no study comparing NACT and NAHT with an HR cut off point of $1 \%$ for advanced breast cancer and we attempted to use a cut-off point of $1 \%$ in this study (13). Our result for HR positive in this population was similar with Cochrane meta-analysis study that also found no difference in overall survival between patients receiving hormonal therapy and chemotherapy (25), but NAHT patients tend to have longer median overall survival than NACT patients. This demonstrates that for advanced breast cancer patients, a $1 \%$ cut-off for HRpositive patients can still be used as a guide to received NAHT when the aim of treatment is palliation. In this study, we did not use trastuzumab as one of the treatments for patients with Her2 positive advanced breast cancer, because currently trastuzumab is not covered for this indication by the national healthcare insurance.

To our knowledge, there is still no study comparing chemotherapy and hormonal therapy in HR-negative breast cancer patients. Standard treatment for HRnegative advanced breast cancer patients is chemotherapy (6). In this study, there were only 3 patients with HR-negative status who received NAHT because they rejected chemotherapy. Median overall survival time of NAHT HR-negative patients was longer than for NACT HR-negative (658 days and 423 days) but not statistically significant and NACT patients have higher risk of death than NAHT patients although patient number was small (HR $4.195 \% \mathrm{Cl} 0.5-30.7$ ).

In this study, after receiving 6 cycles of chemotherapy or 6 months of hormonal therapy, patients would undergo a mastectomy if technically possible. The number of mastectomy patients was almost three times of non-mastectomy patients. Patients who underwent mastectomy after treatment had a statistically significant longer median survival than non-mastectomy patients (Figure $\mathbf{3}$ ). These results were similar to research conducted by Robertson et al., where the life span of patients undergoing mastectomy is better than with tamoxifen alone. Over a 24 month period only $47 \%$ of patients survived when tamoxifen alone was used, versus almost $70 \%$ when mastectomy was performed (26). However, there may have been selection bias since only patients in good condition underwent mastectomy. According to systemic therapy, patients showed better OS and PFS with NAHT than NACT for all of the above characteristics (Figure 4), although some of the results were not statistically meaningful. This suggests that for patients with advanced cancer, chemotherapy has no benefit. It was not similar with Badwe et al. (27), because this study did not only study about advanced breast cancer (4 stage) but also included 3B stage.

The strength of this study is the estimation survival between two arms regardless of HR status. Another strength is related to treatment responses, because there were patients who had HR-negative status but received hormonal therapy. The limitations were the small sample size and the lack of statistical significance in some results. Data distribution was not equal, since this study was not randomized and hence the researcher was less able to control disturbing factors. Another limitation is that we cannot use randomized clinical trial because there was an ethical problem.

\section{CONCLUSIONS}

The oncogenesis process in patients with advanced cancer has been completed. Therefore, the treatment should be palliative and should not worsen the quality of life of the patients. In summary, this study has shown that NAHT as initial treatment for stage $3 B$ and 4 breast cancer offers longer OS than NACT regardless of HR status. The efficacy comparison of these two treatment options warrants further investigation with larger sample size and further exploration of the molecular background.

\section{DECLARATIONS}

\section{Competing of Interest}

The authors declare that they have no competing interests.

\section{Acknowledgement}

We would like to thank all patients in this study and all staffs in Research and Development Department, Dharmais Hospital-National Cancer Center. Authors also acknowledge the support provided by Dharmais Hospital-National Cancer Center for research fund.

\section{REFERENCES}

1. Beatson, GT. On the Treatment of Inoperable Cases of Carcinoma of the Mamma: Sugestions for a New Method of Treatment with Illustrative Cases. Lancet. 1896 Jul 18; ii:104.

2. van de Vijver MJ, He YD, van't Veer LJ, Dai H, Hart AAM, Voskuil DW, et al. A gene-expression signature as a predictor of survival in breast cancer. The New England Journal of Medicine. 2002 Dec 19; 347(25), 1999-2009.

3. EBCTCG. Effects of chemotherapy and hormonal therapy for early breast cancer on recurrence and 15-year survival: An overview of the randomised trials. Lancet. 2005 May 14; 365, 1687-1717. 
4. Cardoso F, Van't Veer LJ, Boogaerts, Slaets G, Viale S, Delalge JY, et al. 70-Gene Signature as an Aid to Treatment Decisions in Early-Stage Breast Cancer. The New England Journal of Medicine.2016 Aug 25; 375(8), 717-29.

5. Asad J, Jacobson AF, Estabrook A, Smith SR, Boolbol SK, Feldman SM, et al. Does oncotype DX recurrence score affect the management of patients with early-stage breast cancer?. The American Journal of Surgery. 2008 Oct; 196(4), 527-9.

6. NCCN clinical practice guidelines in oncology (NCCN guideline): Breast cancer. Version 3; 2015.

7. Teshome M, Hunt K. Neoadjuvant therapy in the treatment of breast cancer. Surg Oncol Clin N Am, 2014 Jul; 23(3), 505-23.

8. Mauriac L, MacGrogan G, Avril A, Durand M, Floquet A, Debled $M$, et al. Neoadjuvant chemotherapy for operable breast carcinoma larger than $3 \mathrm{~cm}$ : a unicentre randomized trial with a 124-month median follow-up. Annals of Oncology. 1999; 10, 47-52.

9. Chia YH, Ellis MJ, Ma CX. Neoadjuvant endocrine therapy in primary breast cancer: indications and use as research tool. British Journal of Cancer. 2010 Aug 10; 103(6), 75964.

10. Bossuyt V, Provenzano E, Symmans WF, Boughey JC, Coles C,Curigliano G, et al. Recommendations for standardized pathological characterization of residual disease for neoadjuvant clinical trials of breast cancer by the BIG-NABCG collaboration. Annals of Oncology. 2015 May 27; 26(7), 1280-91.

11. Chuang E, Christos P, Flam A, McCarville K, Forst M, Shin $S$, et al. Breast cancer subtypes in Asian-Americans differ according Asian ethic group. Journal of Immigrant and Minority Health. 2012 Jan 28; 14, 754-58.

12. American Cancer Society. Breast Cancer Staging 7th Edition. American Joint Committee on Cancer, 1-2. 2010. Available at: cancerstaging.org.

13. Hammond MEH, Hayes DF, Dowsett M, et al. American Society of Clinical Oncology/College of American Pathologists Guideline Recommendations for Immunohistochemical Testing of Estrogen and Progesterone Receptors in Breast Cancer. Archives of pathology \& laboratory medicine. 2010. 134, 907-22.

14. Marcus DM, Switchenko DM, Prabu R, O’Regan R, Zelnak A, Fasola C, et al. Neoadjuvant hormonal therapyis associated with comparable outcomes to neoadjuvant chemotherapy in post-menopausal women with estrogen receptor-positive breast cancer. Frontiers in Oncology. 2013 Dec 27; 3, 1-5.

15. Semiglazov VF, Semiglazov VV, Dahsyan GA, Ziltsova EK, Ivanov VG, Bozhok AA, et al. Phase 2 Randomized Trial of Primary Endocrine Therapy versus Chemotherapy in Postmenopausal Patients with Estrogen Receptor-Positive Breast Cancer. American Cancer Society. 2007 Mar 21; 110(2), 244-54.

16. Preece PE, Wood RA, Mackie CR, Cushieri A. Tamoxifen as initial sole treatment of localized breat cancer in eldelrly women: a pilot study. British Medical Journal. 1982 Mar 20; 284, 869-70.

17. US Department of Health and Human Services, FDA, CDER. Guidance for industry pathological complete response in neoadjuvant treatment of high risk earlystage breast cancer: use as an endpoint to support accelerated approval. New Hampshire Ave. 2014.

18. Rastogi $P$, Anderson SJ, Bear HD, Geyer CE, Kahlenberg MS, Robidoux $A$, et al. Preoperative chemotherapy: updates of national surgical adjuvant breast and bowel project protocols B-18 and B-27. J Clin Oncol. 2008 Feb 10; 26(5), 778-85.

19. Schneeweiss A, Chia S, Hickish T, Harvey V, Eniu Ahegg R, et al. Pertuzumab plus trastuzumab in combination with standard neoadjuvant anthracycline-containing and antrhacycline-free chemotherapy regimens in patients with HER2-positive early breast cancer: a phase II cardiac safety study (TRYPHAENA). Annals of Oncology. $2013 \mathrm{Apr}$ 8; 24(9), 2278-84.

20. Hamy-Petit AS, Belin L, Bonsang-Kitzis $H$, Paquet $C$, Pierga $J Y$, Lerebours $F$, et al. Pathological complete response and prognosis after neoadjuvant chemotherapy for HER2positive breast cancers before and after trastuzumab era: results from a real-life cohort. British Journal of Cancer. 2016; 11, 44-52.

21. Tashiro H, Nomura $Y$, Hisamatsu K (1990). A randomized trial of endocrine therapy, chemotherapy, and chemoendocrine therapy in advanced breast cancer. Gan to Kagaku Ryoho (Japanese Journal of Cancer \& Chemotherapy), 17, 2369-73.

22. von Mickwitz G, Blohmer JU, Costa SD, Denkert C, Eidtman $H$, Eiermann $W$, et al. Response-guided neoadjuvant chemotherapy for breast cancer. Journal of Clinical Oncology. 2013 Oct 10; 31(29), 3623-30.

23. Abe $\mathrm{O}$, Asaishi $\mathrm{K}$, Izuo $\mathrm{M}$, Enomoto $\mathrm{K}$, Koyama $\mathrm{H}$, Tominaga $\mathrm{T}$, et al. Effects of medroxyprogesterone acetate therapy on advanced or recurrent breast cancer and its influences on blood coagulation and the fibrinolytic system. Jpn J Surg. 1995; 25, 701-10.

24. Saad ED, Katz A, Buyse M. Overall Survival and PostProgression Survival in Advanced Breast Cancer: A Review of Recent Randomized Clinical Trials. Journal of Clinical Oncology. 2010 Apr 10; 28(11), 1958-62.

25. Wilcken N, Hornbuckle J, Ghersi D. Chemotherapy alone versus endocrine therapy alone for metastatic breast cancer (Review). The Cochrane Collaboration. 2011. Published by JohnWiley \& Sons, Ltd.

26. Robertson JF, Todd JH, Ellis IO, Elston CW, Blamey RW. Comparison of mastectomy with tamoxifen for treating elderly patients with operable breast cancer. BMJ. 1988; 297, 511.

27. Badwe R, Hawaldar R, Nair N, Parmar V, Siddique S, Budrukkar $A$, et al. Locoregional treatment versus no treatment of the primary tumour in metastatic breast cancer: an open-label randomised controlled trial. Lancet Oncology. 2015 Sep 10; 2045(15), 1-9. 